\title{
High frequency guided wave ultrasound measurements using low-cost equipment
}

\author{
Philip Loveday ${ }^{1 *}$ \\ ${ }^{1}$ School of Mechanical, Industrial and Aeronautical Engineering, University of the Witwatersrand, \\ Johannesburg, Wits 2050, South Africa
}

\begin{abstract}
Guided wave ultrasound can be applied to inspect large volumes of structures from a single transducer location and is particularly effective in one-dimensional structures such as rods, pipes and rail. An application, which is of interest in South Africa, is the inspection rock bolts in the mining industry. The difficulty in inspecting embedded rods is that there is rapid attenuation of the signals with distance. While guided wave systems generally operate at low frequencies $(20 \mathrm{kHz}$ to $200 \mathrm{kHz}$ ), high frequency operation $(1 \mathrm{MHz}$ to $10 \mathrm{MHz})$ can offer some advantages when inspecting rock bolts. Guided wave ultrasound testing is complicated by multi-modal propagation and dispersion, which require the use of sophisticated excitation signals, which are not available in equipment designed for conventional ultrasonic testing. It was therefore necessary to develop a measurement setup and the approach taken was to use standard lab instruments wherever possible and to only purchase the items that are unique to this measurement, using the very limited budget available. The measurement setup was used to perform measurements on a rod and confirmed theoretical predictions. These measurements identified the frequencies where modes propagate with low attenuation and allow the attenuation to be quantified.
\end{abstract}

\section{Introduction}

Guided wave ultrasound offers the possibility of inspecting large volumes of structures from a single transducer location. It is particularly effective in one-dimensional structures such as rods, pipes and rail. This has resulted in inspection and monitoring systems being developed for pipelines in the oil and gas industry internationally [1] and for a heavy haul train line in South Africa [2]. Another application, which has local relevance and interest, is the inspection of rock bolts in the mining industry [3]. The difficulty in inspecting embedded rods is that the ultrasound leaks into the surrounding media causing rapid attenuation of the signals with distance. An instrument for inspecting rock bolts was described in [4], which used high energy, wideband excitation followed by pulse compression digital processing of the received signals to increase the signal to noise ratio. While guided wave systems generally operate at low frequencies $(20 \mathrm{kHz}$ to $200 \mathrm{kHz})$ it was shown by Beard [5] that high frequency operation $(1 \mathrm{MHz}$ to $10 \mathrm{MHz}$ ) can offer some advantages when inspecting

* Corresponding author: Philip.Loveday@wits.ac.za 
embedded structures such as rock bolts and could be combined with the lower frequency measurements. At the higher frequencies, there are some modes of propagation and frequency ranges where the ultrasonic energy is concentrated mainly inside the cross - section of the waveguide with little motion at the surface and this results in reduced leakage of energy.

Guided wave ultrasound testing is more complicated than conventional ultrasonic testing due to multi-modal propagation and dispersion of guided waves. The development of guided wave ultrasound based inspection or monitoring systems requires both numerical modelling capabilities and experimental measurement capabilities. While equipment for conventional ultrasonic testing can operate at high frequencies this equipment is not suitable for guided wave testing, which requires the use of more sophisticated excitation signals. Laboratory equipment for guided wave ultrasound research is available commercially (see for example www.ritecinc.com). A power amplifier for driving a transmit transducer may cost between R100 000 and R 200 000, while a pulser-receiver for pulse-echo measurements could cost between R300 000 and R500 000. Such a system would still require additional instruments such as a two channel arbitrary waveform generator and an oscilloscope and ultrasonic probes and cables. Unfortunately, such a system is not affordable for most South African researchers. This paper aims to show what can be achieved on a very limited budget. The approach taken was to use standard lab instruments wherever possible and to only purchase the items that are unique to this measurement.

Some of the features of guided wave ultrasound are introduced in section 2 as these determine how measurements must be performed and therefore influence the design of the measurement setup. Measurement results obtained from the measurement setup are presented in section 4. The capabilities and limitations of the setup are described in section 5 along with some recommendations for future improvements.

\section{Principles of guided wave ultrasound}

Conventional ultrasonic testing makes use of relatively simple elastic waves that propagate in an infinite medium. Generally, the compressional wave is used although shear waves may also be used. When these waves encounter a free boundary of a structure they reflect and conversion between compressional and shear waves occurs. There are numerous combinations of waves that can satisfy the wave equation inside the structure and the stress free boundary conditions on the perimeter of the waveguide. These solutions are modes that can occur in the waveguide. Some of these modes are travelling waves that propagate and transfer energy along the waveguide. At higher frequencies, where the wavelength is smaller than the cross - section of the waveguide, there are many modes of propagation. These modes of propagation can be dispersive in nature, meaning that energy at different frequencies propagates at different speeds (group velocities). This means that a pulse of energy will become stretched out as it propagates and therefore the amplitude decreases making it more difficult to detect.

Prediction of the guided wave modes and the properties of these modes requires mathematical modelling. Numerical techniques, such as the semi-analytical finite element method, are required for waveguides having complex cross - sections such as a rail. Analytical solutions are available for the cylindrical waveguide (rock bolt) considered in this paper. The dispersion curves for a $20 \mathrm{~mm}$ diameter steel rock bolt are shown in figure 1 . This graph was generated using the free software PCDISP which is described in [6]. This software implements the Pochhammer-Chree solution of the 3D elastic wave equation in a cylinder, which is based on Bessel functions. The final stage of these analytical solutions involves finding the roots of characteristic equations and this only became possible with the arrival of 
digital computers. A Matlab code that implements the analytical solution and finds the roots of the characteristic equation was developed and made available for free by the authors of [6].

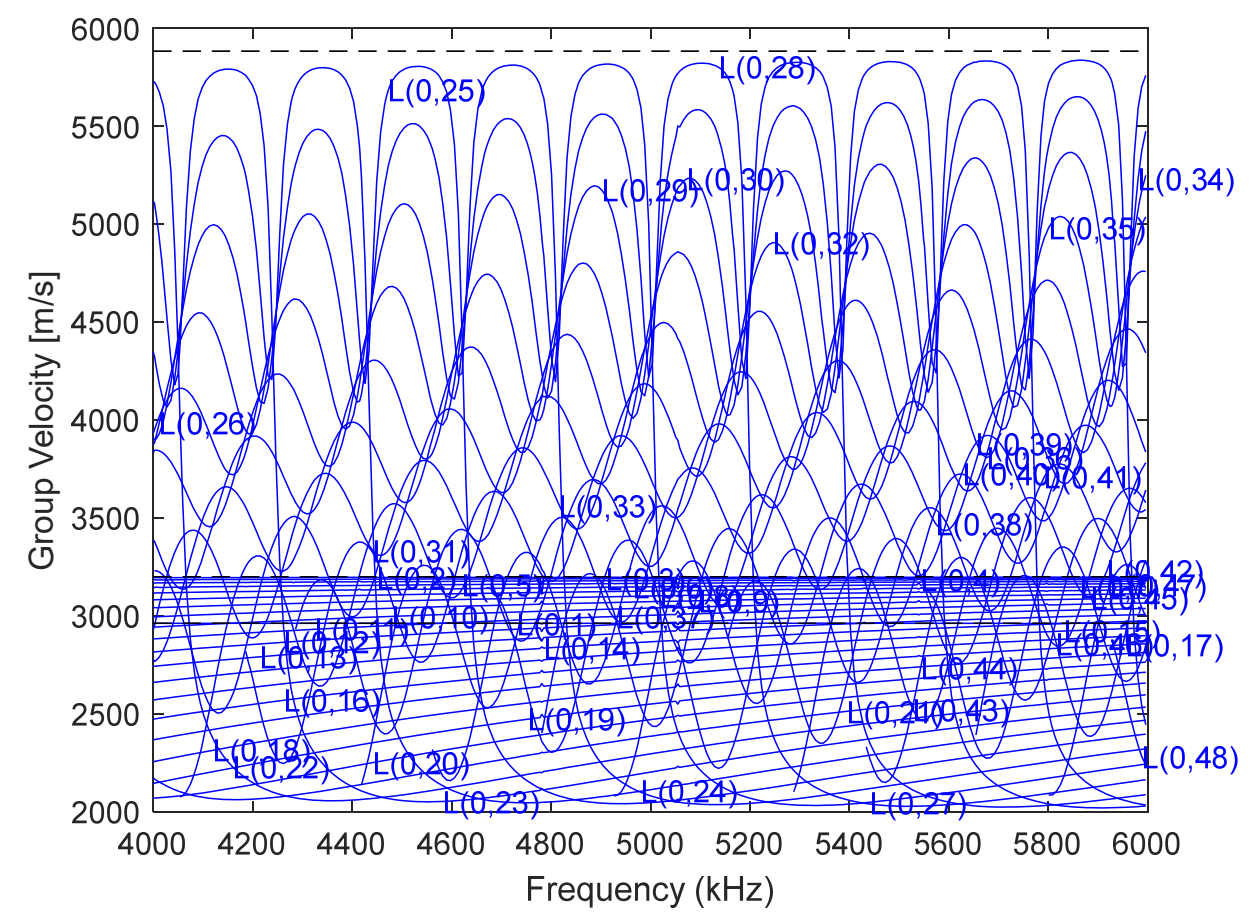

Fig. 1. Group velocity dispersion curves for a $20 \mathrm{~mm}$ diameter steel rod.

The figure shows that at the frequencies being considered there are dozens of modes of propagation. The modes and frequencies where the maximum group velocities are attained are the combinations that are of interest for inspecting embedded rock bolt because it was shown by Beard [5] that these points represent modes where most of the energy is concentrated in the centre of the rock bolt which implies that less energy leaks from the rock bolt into the surrounding medium. Modes and frequencies with higher leakage will attenuate quickly and be filtered out. The points with highest group velocity are also advantageous as energy reflected in these modes will arrive first. Tone burst excitation signals are often used in guided wave ultrasound as these signals have relatively narrow frequency bandwidth for a given length of signal. By increasing the length of the excitation signal (number of cycles) it is possible to reduce the bandwidth of the signal to excite only one mode with a peak group velocity. The tone burst signals are obtained by applying a Hanning window to a sine wave. Example signals with 10 and 50 cycles respectively are shown in figure 2 . These signals were generated by applying the Hanning window to a sine wave with frequency of $5 \mathrm{MHz}$. Figure 2 shows how increasing the number of cycles in the signal causes the bandwidth of the signal to be reduced and the energy at the centre frequency to be increased. Long signals may be used when the distance resolution required is not a concern. There are also other types of signal that could be used including coded signals. It is therefore required that the measurement setup includes the capability to generate arbitrary signals that can be programmed on a computer. 

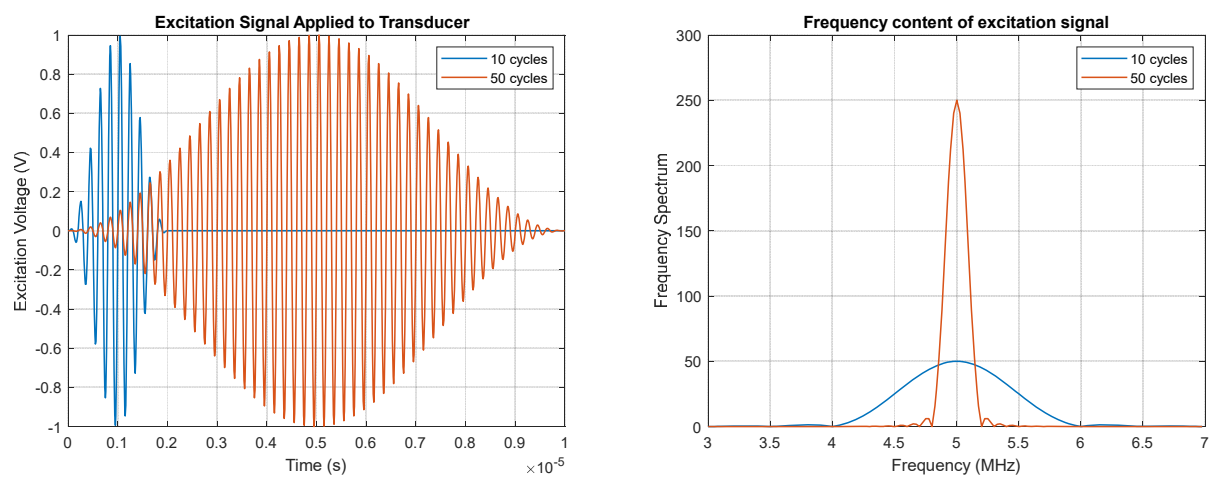

Fig. 2. Tone burst excitation with different signal lengths.

\section{Measurement system}

Measurements may be performed to measure the transmission or reflection of ultrasound. The transmission mode requires the use of two transducers, one to transmit the ultrasound and the second to receive the ultrasound. This mode of measurement is illustrated in figure 3.

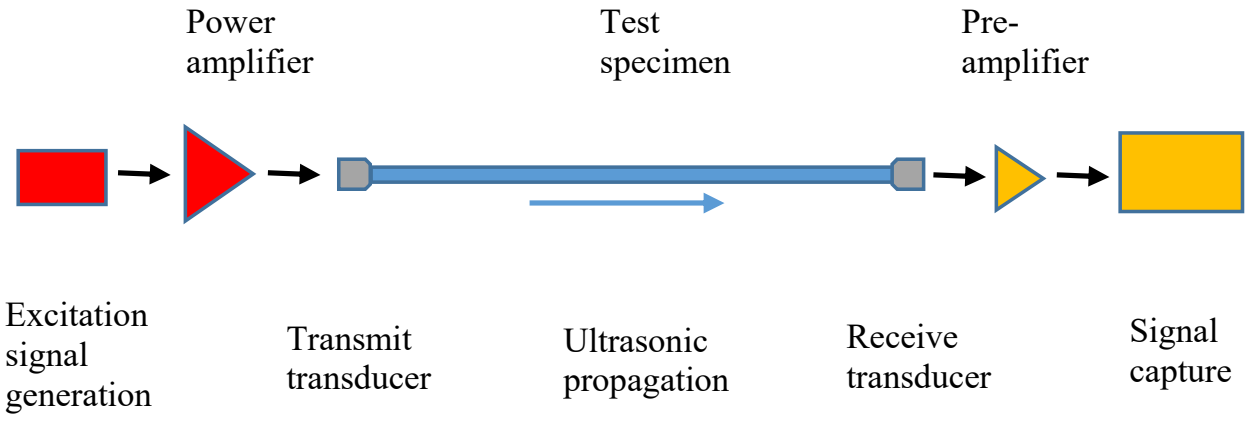

Fig. 3. Transmission mode measurement system configuration.

In the reflection mode a single transducer is used to transmit the ultrasound and then to receive reflected ultrasound. The reflection mode would be required for the inspection of installed rock bolts where only one end of the rock bolt can be accessed. The reflection mode requires additional electronics to protect the receive pre-amplifier from the high voltage excitation signal and then to switch out the power amplifier after the excitation so that this does not cause noise or a low impedance. It was therefore decided to implement the transmission mode measurement first.

The measurement setup is shown in figure 4 and the different components are described in the following subsections. Measurements performed with this system are presented in section 4 . 


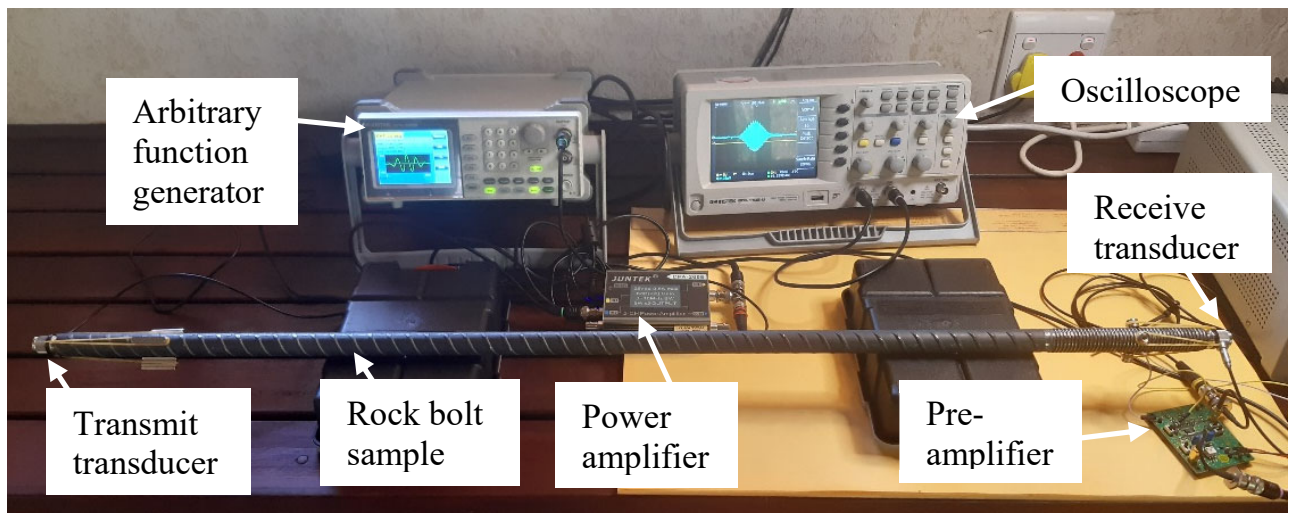

Fig. 4. Measurement system photograph.

\subsection{Piezoelectric ultrasonic transducers}

Two SIUI P5-6L transducers were purchased for this measurement. These are $5 \mathrm{MHz}$ transducers having an active element diameter of $6 \mathrm{~mm}$. The two transducers with cables (BNC to Lemo connectors) and ultrasonic coupling gel cost approximately R8200 (US\$ 600). It is believed that these transducers are relatively inexpensive but have good performance. The transducers were held in place during testing by rubber bands and magnets.

\subsection{Arbitrary function generator}

An arbitrary function generator (GWInstek AFG-2225) was borrowed and used to generate the excitation signals. The signals were programed in Matlab and then saved as a .csv file which was loaded into the memory of the arbitrary function generator. This instrument can output a signal up to 4000 points long, with 10 bit resolution and with a sampling rate up to $120 \mathrm{MSa} / \mathrm{s}$ (mega samples per second). The burst mode was used to output the arbitrary signal and then pause before outputting the signal again.

\subsection{Digital storage oscilloscope}

A digital storage oscilloscope (GWInstek GDS-1102-U) was borrowed and used to capture the signals. This instrument has a $250 \mathrm{MSa} / \mathrm{s}$ real-time sampling rate and a $100 \mathrm{MHz}$ bandwidth. Like almost all digital oscilloscopes this instrument had a vertical resolution of 8 bits and a minimum vertical scale of $2 \mathrm{mV}$ /division. The maximum record length that could be captured was 4000 points, which is low for this type of measurement. This meant that only parts of the received signal could be captured at a time.

\subsection{Power amplifier}

The power amplifier is required to amplify the voltage of the signal produced by the function generator and to supply the current required to drive the piezoelectric transducer. Commercially available power amplifiers for lab measurements in the frequency range of interest tend to be large and expensive. It was therefore necessary to compromise on performance. An alternative was to use a small amplifier that is used to amplify the signals from a function generator. A Juntek DPA-2698 $10 \mathrm{MHz} 2$ channel amplifier was purchased for approximately R1300 from www.banggood.com. This amplifier could only apply a 
maximum excitation amplitude of $25 \mathrm{Vpp}$ to the transducer while it is expected that the transducer could be driven at $100 \mathrm{Vpp}$ or $200 \mathrm{Vpp}$ depending on the duty cycle.

\subsection{Pre-amplifier}

The pre-amplifier is required to amplify the small signal generated by the receive transducer. Again, a good quality ultrasonic pre-amplifier was not affordable. The alternative was to use an evaluation board and a AD8331 Evaluation Board was purchased for approximately R1300. This board is intended for evaluation of the AD8331 IC which is a low noise preamplifier that includes ultrasound as one of the applications. The pre-amplifier board requires a $5 \mathrm{~V}$ DC supply and a power supply (borrowed) was used for this.

\section{Measurement results}

In order to investigate the capabilities of the measurement system a series of measurements were performed on a $1 \mathrm{~m}$ (more accurately, $0.996 \mathrm{~m}$ ) long portion of a rock bolt, which had a diameter of $20 \mathrm{~mm}$.

The first measurement was performed using a 10 cycle tone burst signal with a centre frequency of $5 \mathrm{MHz}$. This is a fairly large bandwidth signal (see figure 2) having energy spread across the peak velocities of numerous modes shown in figure 1 . The pre-amplifier was not used in this measurement. The received signal is shown in figure 5. The first figure shows the entire captured signal while the second figure shows a zoomed in view of part of the signal. The first wave packet arrived at approximately $0.17 \mathrm{~ms}$ and there appear to be a succession of wave packets following the first one. It is tempting to think that the different wave packets observed correspond to different modes of propagation each having different group velocities. This is actually not the case as the peak group velocities are all very similar (less than $1 \%$ difference). Instead, this phenomenon is due to the presence of numerous modes propagating with dispersion causing the wave packets to become stretched in time. These different modes interfere constructively in certain time intervals and destructively in other time intervals and this causes the signal to appear like a succession of wave packets. This phenomenon was described by Beard [5] who showed that if the signal is filtered to contain only the frequency range where one mode has a peak group velocity (for example 4.8 $\mathrm{MHz}$ to $5 \mathrm{MHz}$ ) then the response is that of the single mode with dispersion. The frequency spectrum of the signal is shown in figure 6 . It is seen that there is more energy in the frequency bands corresponding to the peak group velocities shown in figure 1.
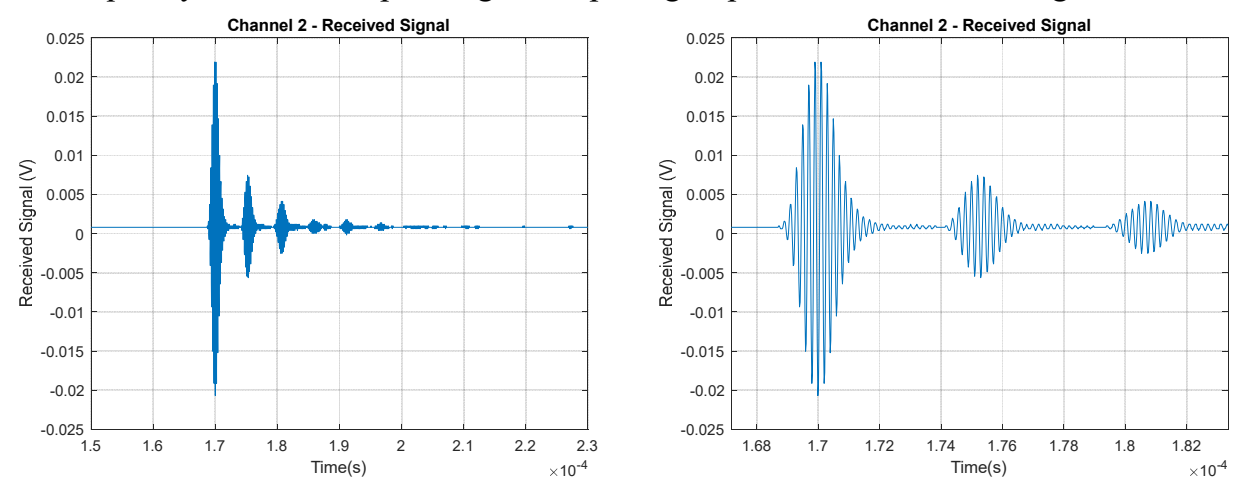

Fig. 5. Received signal for a 10 cycle tone burst with centre frequency of $5 \mathrm{MHz}$. 


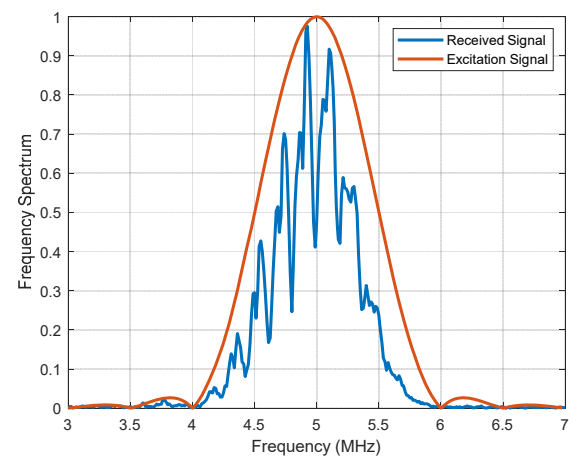

Fig. 6. Frequency spectrum of received signal and excitation signal.

This first result illustrates that in guided wave ultrasound the multi-modal nature of the propagation can make interpretation of the results complicated. To excite or sense individual modes, use is sometimes made of transducer arrays in which the spacing of the elements and phasing of the excitation signals is selected to preferentially excite a particular mode. A second option, which is more appropriate here, is to reduce the frequency bandwidth of the excitation signal. This can be achieved by increasing the number of cycles in the excitation tone burst signal. A tone burst signal containing 200 cycles was used for the remainder of the tests.

The centre frequency of the excitation signal was varied and the frequencies were maximum receive responses were obtained were recorded. Table 1 lists the peak group velocities and the frequencies at which these were predicted from the model. The measured frequencies, where peak amplitude responses were observed are compared to these frequencies. It is seen that there is generally very good agreement between the predicted and measured frequencies. This provides a simple way to measure these frequencies. These are the candidate frequencies where long range testing could be performed.

Table 1. Predicted peak velocity frequencies and measured peak amplitude frequencies

\begin{tabular}{|l|l|l|l|l|l|l|l|l|l|}
\hline Mode & L0,24 & L0,25 & L0,26 & L0,27 & L0,28 & L0,29 & L0,30 & L0,31 & L0,32 \\
\hline $\begin{array}{l}\text { Predicted } \\
\text { Peak Group } \\
\begin{array}{l}\text { Velocity } \\
\text { (m/s) }\end{array}\end{array}$ & 5800 & 5806 & 5812 & 5818 & 5822 & 5827 & 5830 & 5834 & 5838 \\
\hline $\begin{array}{l}\text { Predicted } \\
\text { Peak GV } \\
\text { Frequency } \\
\text { (MHz) }\end{array}$ & 4.340 & 4.535 & 4.723 & 4.910 & 5.105 & 5.293 & 5.480 & 5.676 & 5.863 \\
\hline $\begin{array}{l}\text { Measured } \\
\text { Peak Amp } \\
\text { Frequency } \\
\text { (MHz) }\end{array}$ & 4.360 & 4.550 & 4.735 & 4.925 & 5.110 & 5.290 & 5.470 & 5.640 & 5.835 \\
\hline
\end{tabular}

Next we investigate the signals obtained from multiple arrivals after multiple reflections. For these measurements a 200 cycle tone burst excitation signal with centre frequency of $4.925 \mathrm{MHz}$ was used. The first arrival corresponds to $1 \mathrm{~m}$ of travel and took approximately $0.17 \mathrm{~ms}$ to arrive. The second and third arrivals correspond to $3 \mathrm{~m}$ and $5 \mathrm{~m}$ of travel and arrive at proportionally later times. The first three arrivals are shown in figure 7 . If the oscilloscope had more memory it would be possible to show all the arrivals in one captured 
signal. The time between arrivals can be used to estimate the group velocity while the decrease in amplitude can be used to estimate the attenuation. In this measurement the successive arrivals appear to be approximately 10 times smaller $(-20 \mathrm{~dB})$ and therefore the attenuation per metre of wave propagation is approximately $-10 \mathrm{~dB} / \mathrm{m}$.
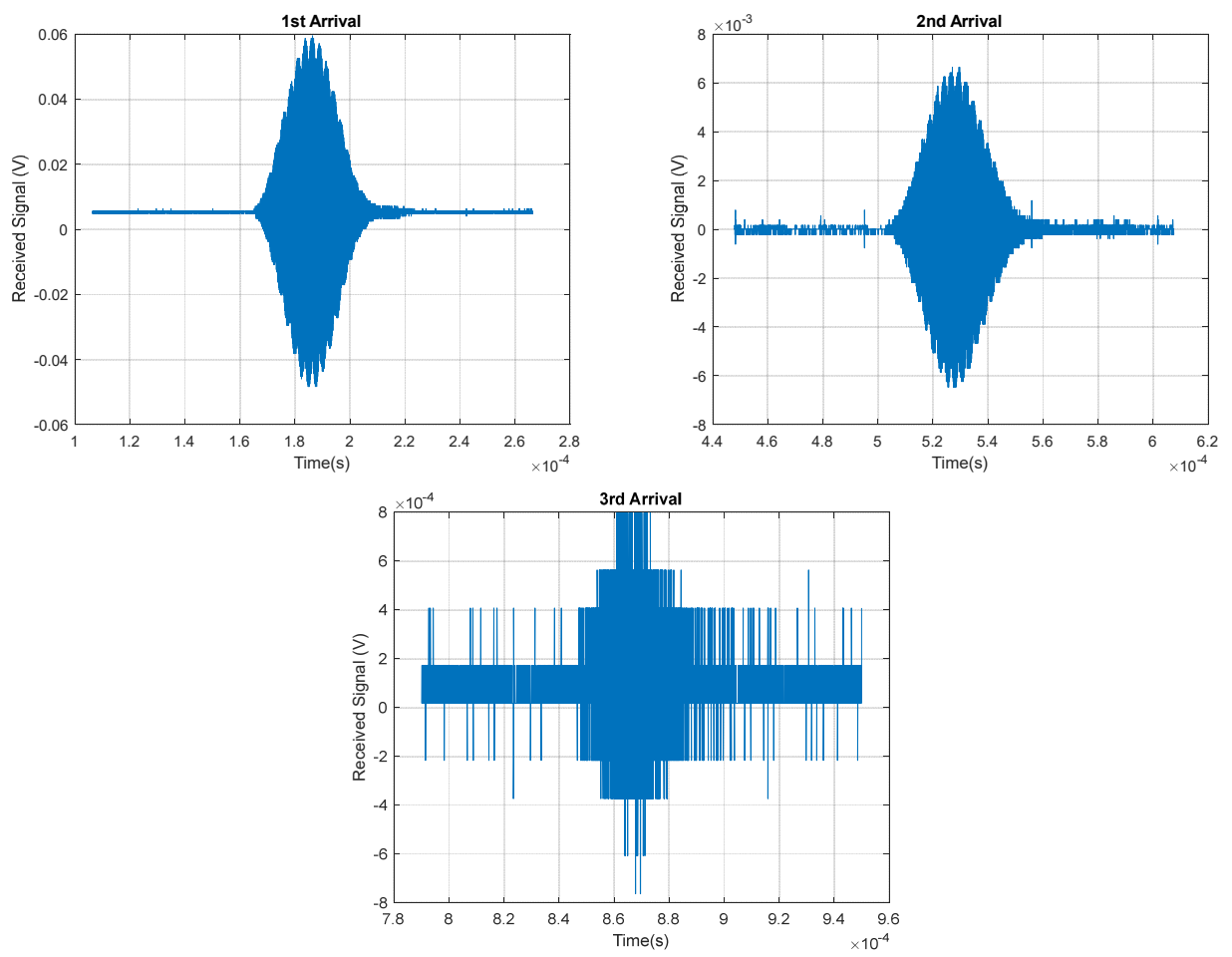

Fig. 7. First three arrivals.

It can be seen that the third arrival signal is very small and discrete levels are seen in the signal. This occurs because the oscilloscope is set on the smallest vertical scale of $2 \mathrm{mV} / \mathrm{div}$ and has only 8 bit resolution. In order to measure these small signals more accurately it was necessary to use the pre-amplifier. Figure 8 shows the same arrival but with the pre-amplifier used to amplify the received signal. This signal was measured using a vertical scale of 10 $\mathrm{mV} / \mathrm{div}$ and is shown in the first graph in figure 8. The voltage signal is approximately 40 times larger than the signal without the pre-amplifier (third graph in figure 7). There is also considerable noise on this signal, which can be reduced by averaging as illustrated in the second and third graphs in figure 8 where 16 and 256 averages were used respectively. This averaging was performed on the oscilloscope. When random noise is present in a signal averaging may be applied to decrease the random noise without reducing the actual signal. The reduction in noise is related to the square root of the number of averages. Therefore 16 and 256 averages should reduce the noise to $1 / 4^{\text {th }}$ and $1 / 16^{\text {th }}$ of what is present in one measurement respectively. 

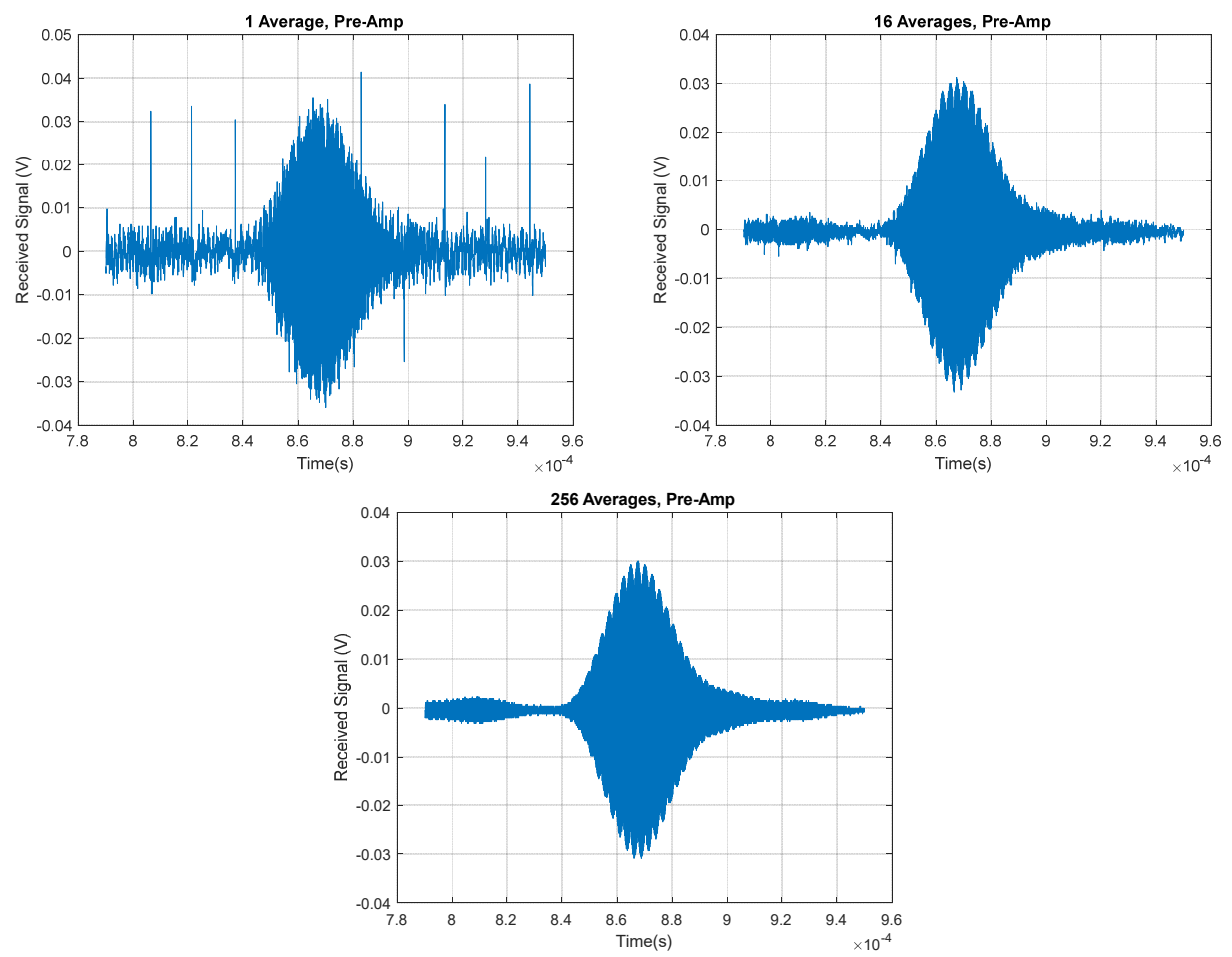

Fig. 8. Third arrival measured with pre-amplifier and averaging.

With this measurement setup it was possible to measure two more arrivals. The fourth and fifth arrivals shown in figure 9 involve propagation of the wave over distances of $7 \mathrm{~m}$ and $9 \mathrm{~m}$ respectively. Again it is observed that the signals decrease by a factor of ten for each additional arrival. The fifth arrival signal is very small and was the smallest signal that could be observed.
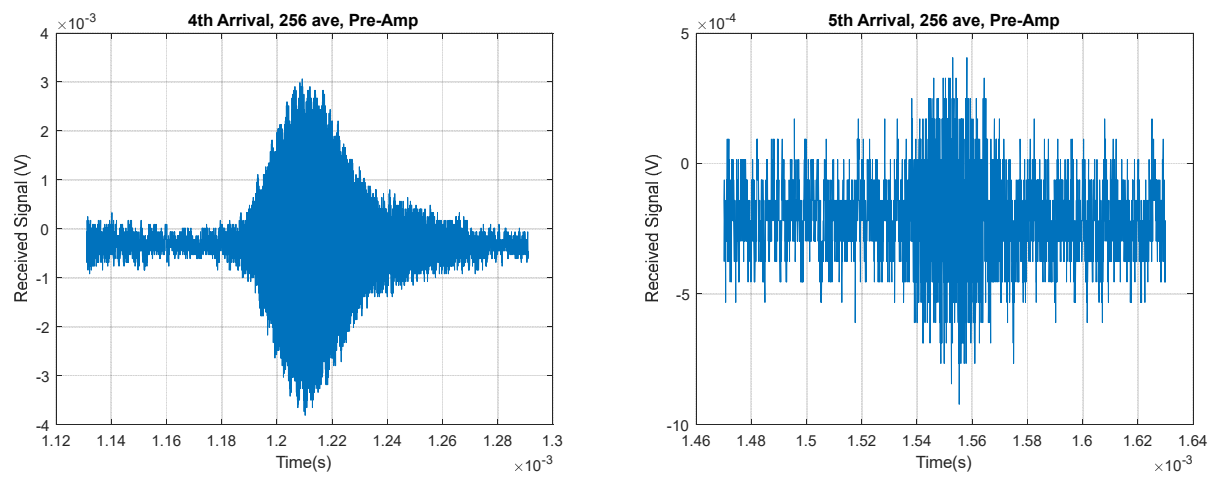

Fig. 9. 4th and 5th arrivals measured with pre-amplifier and averaging.

Once the signals have been captured and transferred to a computer it is possible to perform post processing. The signal has energy only in a small frequency range and if only frequencies between 4.8 and $5 \mathrm{MHz}$ are retained then the signal becomes clearer as shown in figure 10 . 


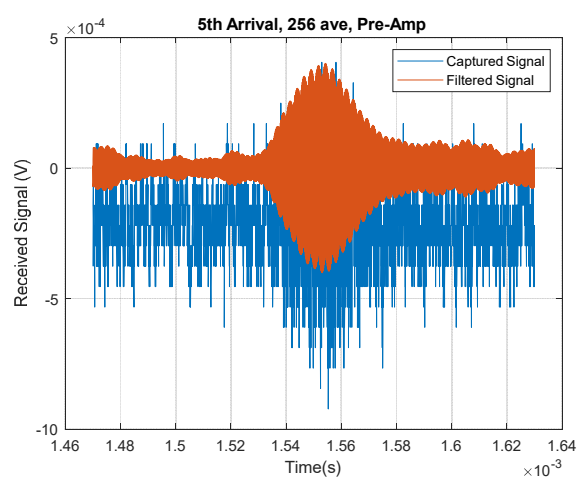

Fig. 10. Filtering to reduce noise.

\section{Conclusions and future work}

The system is capable of performing high frequency guided wave ultrasound measurements. Measurements were performed on a section of a rock bolt between 4 and $6 \mathrm{MHz}$ and various guided wave modes were detected in good agreement with theoretical predictions. A wave that had propagated a total distance of $9 \mathrm{~m}$ in the rock bolt could be detected. The system can now be used, for example, to quantify the additional attenuation introduced when the rock bolt is embedded in concrete.

The measurement system was created using borrowed lab instruments and a few relatively inexpensive purchases. The total cost of the power amplifier, pre-amplifier, two transducers and cables was approximately R11000. The value of the borrowed instruments would be around R20 000. The function generator and the oscilloscope had limited memory and this restriction will be removed by purchasing new instruments which will cost a total of R22000 bringing the total cost of the system to R33 000 (US\$ 2200 ). High frequency guided wave ultrasound measurements can therefore be performed on a limited budget.

PC based oscilloscopes were considered as an alternative to the bench top instruments. A product that met all the performance requirements and had a significant cost saving was not identified and the ease of use of bench top instruments was preferred. PC based oscilloscopes may be reconsidered at a later stage if field measurements are to be performed.

The drive amplifier does not supply the maximum voltage levels that can be used and this could be improved. Current efforts in this area involve the development of a MOSFET based amplifier. Reflection mode testing should be implemented. This requires additional electronics and these are being developed.

\section{References}

1. P. Cawley, Struct. Heal. Monit. 17, 1225-1244 (2018)

2. P.W. Loveday, J. Nondestruct. Eval. 31, 303-309 (2012)

3. B.J. Buys, P.S. Heyns, P.W. Loveday, J. South. Afr. Inst. Min. Metall. 109, 2 (2009)

4. T. Stepinski, K. Mattson, K, Instrument for Rock Bolt Inspection by Means of Ultrasound, in IEEE International Ultrasonics Symposium Proceedings, pp. 4-7 (2015)

5. M.D. Beard, Guided Wave Inspection of Embedded Cylindrical Structures, $\mathrm{PhD}$ Thesis, Imperial College (2002)

6. F. Seco, AR. Jiménez, Modelling the Generation and Propagation of Ultrasonic Signals in Cylindrical Waveguides. In Ultrasonic Waves, Dr Santos (Ed.), pp. 1-28 (2012) 